\title{
Tsafon
}

Revue d'études juives du Nord

$82 \mid 2021$

Enjeux esthétiques dans la littérature après Auschwitz

\section{Bande Alexandre, Biscarat Pierre-Jérôme et Lalieu Olivier, Nouvelle histoire de la Shoah}

Danielle Delmaire

\section{(2) OpenEdition}

1 Journals

Édition électronique

URL : https://journals.openedition.org/tsafon/4517

DOI : $10.4000 /$ tsafon. 4517

ISSN : 2609-6420

Éditeur

Association Jean-Marie Delmaire

Édition imprimée

Date de publication : 1 décembre 2021

Pagination : 154-158

ISSN : 1149-6630

\section{Référence électronique}

Danielle Delmaire, « Bande Alexandre, Biscarat Pierre-Jérôme et Lalieu Olivier, Nouvelle histoire de la Shoah », Tsafon [En ligne], 82 | 2021, mis en ligne le 01 décembre 2021, consulté le 12 février 2022 URL : http://journals.openedition.org/tsafon/4517 ; DOI : https://doi.org/10.4000/tsafon.4517

Ce document a été généré automatiquement le 12 février 2022.

Tsafon. Revues d'études juives du Nord 


\title{
Bande Alexandre, Biscarat Pierre- Jérôme et Lalieu Olivier, Nouvelle histoire de la Shoah
}

\author{
Danielle Delmaire
}

\section{RÉFÉRENCE}

Bande Alexandre, Biscarat Pierre-Jérôme et Lalieu Olivier (dirigé par), Nouvelle histoire

de la Shoah, Paris, Passés composés, août 2021, 413 p. $24 €$.

1 Vingt contributions composent ce volume dont le titre annonce une réécriture de l'histoire de la Shoah, en France et dans toute l'Europe et même de la persécution des Tsiganes puisque Ilsen About présente le "génocide et [les] persécutions des Roms et des Sinti en Europe 1933-1946 ». Elles ont été rassemblées par des historiens confirmés de la Shoah : Alexandre Bande est expert auprès de la Fondation pour la Mémoire de la Shoah et intervient au Mémorial de la Shoah, Pierre-Jérôme Biscarat fut responsable pédagogique pour la Maison d'Izieu, mémorial des enfants juifs exterminés, et a travaillé jusqu'en 2020 avec l'association Yahad-In Unum qui met en évidence la Shoah par balles dans l'Europe orientale, Olivier Lalieu, auteur de plusieurs ouvrages sur la mémoire de la Shoah, est responsable de l'aménagement des lieux de mémoire et des projets externes du Mémorial de la Shoah et membre du Centre international pour l'éducation sur Auschwitz et l'Holocauste au musée d'État d'Auschwitz-Birkenau. L'ouvrage est dédié à Serge et Beate Klarsfeld qui ont «inspiré, guidé et soutenu » les travaux de ces historiens.

2 La vingtaine d'études présentée dans cet épais volume de plus de 400 pages couvre toute l'Europe, soit tout l'espace de la mise en œuvre de la Shoah, et certaines d'entre elles se placent dans la perspective d'autres génocides. Elles se répartissent en quatre parties.

3 Première partie : Origines, étapes et géographie du processus génocidaire. 
4 Elle expose le contexte particulièrement en Europe centrale et orientale. Johan Puttemans situe l'origine du processus génocidaire dans l'extermination, dès la fin de la décennie 1930, des handicapés physiques et mentaux. L'auteur renouvelle effectivement la compréhension des meurtres collectifs en soulignant le lien entre extermination des handicapés et disparition des Juifs et des Tsiganes, jugés eux aussi asociaux et « inutiles ». Les procédés sont communs : chambres à gaz, injections létales effectuées secrètement, les traces doivent aussi disparaître.

5 Il revenait à Tal Bruttmann d'étudier la "mise en place de la 'Solution finale' ». Il insiste sur le «tournant de l'été 1941 ». Trop souvent l'origine de l'extermination des Juifs est datée avec la conférence de Wannsee (20 janvier 1942) mais les tueries de Juifs par les Einsaztgruppen, la mise à mort des prétendus «judéo-bolcheviques», les pogromes en Europe (Jedwabne) etc. sont antérieurs. «À la mi-septembre 1941, Hitler ordonne que le territoire de l'Allemagne soit débarrassé de toute présence juive » (p. 40). L'article est remarquable pour ses notes abondantes et largement historiographiques.

6 Les trois articles suivants présentent les procédés de l'extermination: la mise à mort dans les ghettos (Audrey Kichelewski), les tueries de masse avec la « Shoah par balles » (Andrej Umansky et Patrick Desbois), les «centres de mise à mort» (Christophe Tarricone). La carte couverte de points figurant les ghettos (p. 50) montre que ces structures de concentration humaine furent excessivement nombreuses et, finalement, d'une grande diversité. D'où l'importance de définir les termes car quels points communs unissent le ghetto de Varsovie et le quartier de Salonique réservé aux Juifs, appelé aussi ghetto et qui fut plutôt un camp de transit? Les conditions de vie ou de survie varient selon les lieux d'implantation et les époques. De même Christophe Tarricone s'attache à définir le terme de «centre de mise à mort » qui « rend compte du sort spécifique des Juifs» dans les pratiques répressives des nazis. Ce centre peut être une chambre à gaz ou bien le lieu d'une fusillade de masse comme à Ponar, près de Vilna. Il peut évoluer aussi avec les mois pour devenir un camp entouré de barbelés et, dans un souci de rationaliser la mise à mort, ce camp est toujours installé près des routes et des voies ferrées. Ici, comme souvent ailleurs, la discrétion impose la destruction des corps. Après quinze années de prospection en Europe orientale, Patrick Desbois et son équipe est parvenue à définir cinq étapes dans le déroulement d'une exécution par balles: le rassemblement des victimes, leur déplacement, leur déshabillage, la fusillade et enfin le dépouillement. Là encore, les assassins effacent les traces autant pour masquer les crimes que pour tromper le voisinage qui pourtant parfois participe à la curée. Il faut remercier Umansky et Desbois pour l'exposé de leur méthodologie, dans leurs notes.

7 Avec la Pologne, les territoires soviétiques furent particulièrement touchés par les massacres mais la prise de conscience du danger par les victimes elles-mêmes est à l'origine des sauvetages, sujet étudié par Marie Moutier-Bitan. L'auteure dresse la carte des lieux de sauvetage car si les Juifs prennent eux-mêmes la décision de se cacher, ils ont besoin d'aides pour survivre, même dans les forêts. Les sauvetages ne réussissent pas toujours et les échecs sont parfois occultés par l'image du Juste sauveteur.

8 Cette première partie se clôt par une heureuse contribution de Ilsen About sur le génocide des Roms et des Sinti qui commence dès 1933. Parfois « des Roms partagent le sort des Juifs dans des ghettos ou dans des camps » (p. 134), dans les territoires conquis par les Allemands. Les massacres et les asphyxies par le gaz sont le lot commun. 
T

des Juifs dans la résistance à la politique d'extermination dans la mesure où ses références bibliographiques se cantonnent à la fin du siècle dernier. Il analyse la présence des Juifs dans les différents mouvements de résistance, dans les maquis (juifs parfois), dans les rangs gaullistes etc. Quant à Cindy Biesse qui brosse un tableau de l'aide et des sauvetages accordés aux Juifs, elle a raison de diviser la chronologie en un avant et un après l'été 1942 durant lequel les rafles sont les plus nombreuses. Elle rappelle, à juste titre, l'engagement des mouvements non juifs comme la CIMADE et juifs comme l'OSE, les Éclaireurs israélites de France, le Joint. Elle élabore une sociologie des sauveteurs, comme a pu le faire Jacques Sémelin ${ }^{1}$, et pointe l'importance des cheminots pour évoquer leur implication dans le sauvetage des Juifs en gare de Lille-Fives mais ses informations sont maladroites et jamais ces cheminots ne sont parvenus «à extraire une centaine d'enfants des wagons à destination des centres de mise à mort » (p. 207). En revanche ils ont accompli l'exploit de sauver une soixantaine de personnes, adultes et enfants ${ }^{2}$.

11 Laurent Joly fait le point sur la politique du Gouvernement de Vichy envers les Juifs et liste toutes les décisions: statuts, recensements, "aryanisation» etc. Il établit le parallèle entre la politique allemande et la politique de Vichy pour conclure qu'elles se complètent parfaitement. À propos de la rafle du Vel d'Hiv, il rappelle qu'elle fut globalement un échec dans la mesure où, dans la grande majorité des arrondissements de Paris, le taux d'arrestation est inférieur à $50 \%$ du nombre des personnes à arrêter. Et il invite, comme le font la plupart des historiens maintenant, à en finir avec « le cliché des victimes passives ", car si la rafle échoue c'est que les Juifs lui résiste en se cachant.

Thomas Fontaine apporte une chronologie, bien établie désormais grâce aux travaux récents dont il fait état dans ses notes abondantes, de la déportation des Juifs de France. La première étape touche les Juifs communistes, déportés par représailles ${ }^{3}$, puis vient le tournant de juin et juillet 1942 avec les grandes rafles responsables du surpeuplement des camps de rassemblement. C'est durant l'été 1942, se prolongeant jusqu'à novembre, qu'est réalisée plus de la moitié du programme de la « Solution finale » en France. Par la suite, la machine s'enraye et les convois sont de plus en plus espacés. L'auteur insiste sur les moyens de la déportation: les voies ferrées, les gares et la réquisition du personnel de la SNCF.

C'est, me semble-t-il, la partie qui apporte le plus de nouveautés, en outre fort utiles aux enseignants. Quatre chapitres, tous de bonne facture suffisent. Olivier Lalieu déroule les expressions de la mémoire qui dès 1945 se sont concrétisées par l'apposition de plaques, par l'érection de monuments, par des manifestations publiques, par des œuvres littéraires ou cinématographiques etc. La voix était accordée aux victimes. Mais la mémoire de la déportation raciale se perdait dans la mémoire de la déportation en générale qui pendant de longues années occulta la particularité de la persécution des Juifs. La confusion perdura et pourtant les chiffres sont là pour mettre en évidence la différence entre les déportés politiques et les déportés victimes du racisme: les premiers revinrent dix fois plus nombreux que les seconds qui en outre comptaient des enfants, des vieillards. Et à leur retour, les déportés étaient noyés dans le grand nombre des prisonniers de guerre (950 000 prisonniers). Les Juifs étaient une « minorité dans la 
minorité » et « des victimes de second rang ». Il y eut bien des associations de déportés mais elles regroupaient trop souvent tous les déportés ${ }^{4}$. Le procès Eichman, à Jérusalem en 1961, éveilla les consciences sur la particularité de la Shoah puis divers événements contribuèrent à faire la différence : la fondation de l'association des Fils et Filles de Déportés de Serge et Beate Klarsfeld (1979), le discours du président Chirac (1995), la Fondation pour la Mémoire de la Shoah (2000) et des œuvres cinématographiques marquèrent les esprits (la série Holocauste et le film Shoah de Claude Lanzman).

«Les voyages de mémoire sur les lieux de la Shoah » sont analysés par Alexandre Bande et Pierre-Jérôme Biscarat, tous deux engagés dans des démarches pédagogiques pour transmettre la mémoire de la Shoah. Ils s'accordent pour souligner l'importance d'une visualisation de la persécution. Il ne s'agit pas toujours de visiter un camp comme celui d'Auschwitz, les élèves peuvent être amenés sur un tout autre lieu de mémoire, comme la Maison d'Izieu où P-J Biscarat exerça de nombreuses années : une démarche dont les retombées s'avèrent très positives pour la transmission de la mémoire de la Shoah. Citons encore parmi bien d'autres lieux : Drancy (et son wagon du souvenir), Beaune-laRolande, Pithiviers, Gurs, Rivesaltes etc ${ }^{5}$. Enfin des souvenirs de déportés et des œuvres littéraires comme celles de l'écrivain Aharon Appelfeld sont aussi autant de lieux de mémoire à grande valeur pédagogique.

Suite à cet article véritable guide pour l'enseignement de la mémoire de la Shoah, Ygal Fijalkow et Jacques Fijalkow interrogent sur les effets que produisent les visites scolaires d'Auschwitz-Birkenau. Constatant que ces visites sont de plus en plus nombreuses, ces deux dernières décennies, les auteurs s'attachent à donner quelques conseils aux enseignants, insistant sur l'indispensable préparation car l'émotion ne doit pas être le but premier voyage. Celui-ci doit éveiller la conscience politique du visiteur, éradiquer ses préjugés sur le racisme et l'antisémitisme. La visite n'est pas touristique mais elle doit faire percevoir des valeurs morales. Et finalement, il revient à chaque enseignant d'ajuster la préparation à son public car «toutes les visites ne se ressemblent pas » (p. 264).

17 Au terme de cette partie, Iannis Roder, enseignant d'histoire dans un collège de la banlieue parisienne et attaché au Mémorial de la Shoah, expose «Les défis de l'enseignement de la Shoah ». L'article offre une aide fort utile aux enseignants afin de surmonter les difficultés qu'ils peuvent rencontrer en présentant la persécution raciale. Il insiste sur la nécessité de montrer une histoire positive des Juifs et éviter d'en faire les éternelles victimes d'une fatalité inévitable. L'éducation à la citoyenneté doit contrer cette fatalité. Les témoins peuvent abonder dans ce sens en évoquant leur parcours de déportés mais aussi de résistants. Et surtout, l'auteur conseille d'adapter l'enseignement à son public d'élèves « car chaque âge ne peut être soumis aux mêmes images » (p. 276).

Quatrième partie : Questions sensibles.

19 Cinq questions sensibles sont étudiées, souvent appartenant à l'actualité. Trois d'entre elles ne sont pas apparues récemment: la connaissance des Alliés quant à l'extermination des Juifs (Willy Coutin), les rapports entre le monde arabe et l'Allemagne nazie (Dominique Trimbur) et le négationnisme de 1948 à 2021 (Valérie Igounet) sont des questions que ces trois auteurs ont d'ailleurs déjà travaillées et les trois bibliographies citent des ouvrages très récents mais aussi plus anciens des années 1980. 

d'information étaient multiples, vers la Grande-Bretagne, les USA et l'URSS. Il rappelle le rôle, vers l'Ouest, de Ian Karski, désormais bien connu, ainsi que les révélations provenant du Bund, du ghetto de Varsovie par Ringelblum ou encore du Joint. Mais la priorité des Alliés était de gagner la guerre et le gouvernement à Moscou restait silencieux. connue également mais Dominique Trimbur élabore, fort heureusement, un état de la question et en donne une fine étude historiographique. Puis il évoque quelques paradoxes. Dès la fin du XIX ${ }^{e}$ siècle l'Allemagne portait son intérêt sur la Palestine et le Moyen-Orient et s'intéressait au sionisme. Hitler considérait les Sémites comme une race inférieure, ce qui n'empêcha pas des nationalistes arabes opposés au sionisme d'adhérer à son idéologie. En revanche des musulmans ont été reconnus Justes parmi les Nations pour avoir aidé des Juifs, surtout en Albanie. Question complexe s'il en est.

Quant à l'histoire du négationnisme, Valérie Igounet rappelle que ce fut une invention française dès le lendemain de la guerre (Bardèche et Rassinier). Puis la Guerre des Six Jours eut pour conséquence la dénonciation de l'impérialisme juif. Enfin vint Faurisson qui dès les années 1980 affirmait que l'histoire de la Shoah était falsifiée. D’autres noms apparaissent: Noam Chomsky, Roques, J-G Cohn-Bendit, Garaudy et le succès du négationnisme auprès de Le Pen. Enfin la seconde Intifada des années 2000 n'a fait qu'amplifier le courant (Dieudonné, Soral).

23 Avec ces trois questions, une quatrième s'impose: le complotisme associé à l'antisémitisme étudié par le spécialiste de la question : Rudy Reichstadt. C'est une idée ancienne qui refait actuellement surface. Lors des dernières manifestations de rue, en France, la puissance des Rothschild est à nouveau dénoncée, le sionisme est satanique et le droit des Juifs à un État est nié.

Spécialiste du génocide arménien, Yves Ternon interroge: "Peut-on comparer les génocides? » puisque désormais il est reconnu que d'autres génocides ont précédé et suivi celui des Juifs (Hereros, famine en Ukraine, Rwanda, Kmers rouges). La réponse varie selon qu'elle provient du juriste qui tient compte du droit international ou de l'historien qui lui peut dégager trois temps dans le processus génocidaire : la définition d'un groupe supérieur, la mise en place d'un pouvoir totalitaire gangréné par une phobie paranoïaque, l'élaboration de diverses techniques d'assassinats.

Il faut espérer que cet ouvrage parvienne à bénéficier d'un large lectorat notamment parmi les historiens travaillant sur la Shoah mais aussi et surtout parmi les enseignants que la troisième partie concerne particulièrement.

Quelques regrets : l'absence d'index et d'une bibliographie générale qui complèterait les bibliographies qui suivent chaque article ; l'inégalité des articles. Certains se placent à la pointe de la connaissance avec une bibliographie et une historiographie bien développées qui justifient le titre de l'ouvrage, d'autres se limitent à une historiographie datée ou trop générale faisant l'impasse sur des études plus fines. 


\section{NOTES}

1. Parmi ses ouvrages : La survie des juifs en France (1940-1944), Paris, CNRS éditions, 2018.

2. L'article de Monique Heddebaut paru dans notre $n^{\circ} 70$, automne 2015-hiver 2016, « Sans armes face à la rafle du 11 septembre 1942, dans la Zone rattachée à Bruxelles », p 119-178, non mentionné, aurait été utile.

3. Il en est de même dans le nord de la France soumis au commandement militaire de Bruxelles.

4. Dès 1955, Lili Leignel petite fille juive déportée de Roubaix à Ravensbrück, à l'âge de 11 ans, témoignait dans le cadre d'une association d'anciens résistants.

5. Ajoutons pour les Hauts-de-France : la caserne Dossin à Malines ou les parcours de mémoire mis en place dans quelques villes du Nord et du Pas-de-Calais avec le soutien du Mémorial de la Shoah et l'Inspection académique. 